\title{
Browder's Convergence for Uniformly Asymptotically Regular Nonexpansive Semigroups in Hilbert Spaces
}

\author{
Genaro López Acedo ${ }^{1}$ and Tomonari Suzuki ${ }^{\mathbf{2}}$ \\ ${ }^{1}$ Departamento de Análisis Matemático, Facultad de Matemáticas, Universidad de Sevilla, \\ 41080 Sevilla, Spain \\ ${ }^{2}$ Department of Mathematics, Kyushu Institute of Technology, Tobata, Kitakyushu 804-8550, Japan \\ Correspondence should be addressed to Genaro López Acedo, glopez@us.es \\ Received 6 October 2009; Accepted 14 October 2009 \\ Academic Editor: Tomas Dominguez Benavides
}

Copyright (C) 2010 G. López Acedo and T. Suzuki. This is an open access article distributed under the Creative Commons Attribution License, which permits unrestricted use, distribution, and reproduction in any medium, provided the original work is properly cited.

We give a sufficient and necessary condition concerning a Browder's convergence type theorem for uniformly asymptotically regular one-parameter nonexpansive semigroups in Hilbert spaces.

\section{Introduction}

Let $C$ be a closed convex subset of a Hilbert space $E$. A mapping $T$ on $C$ is called a nonexpansive mapping if $\|T x-T y\| \leq\|x-y\|$ for all $x, y \in C$. We denote by $F(T)$ the set of fixed points of $T$. Browder, see [1], proved that $F(T)$ is nonempty provided that $C$ is, in addition, bounded. Kirk in a very celebrated paper, see [2], extended this result to the setting of reflexive Banach spaces with normal structure.

Browder [3] initiated the investigation of an implicit method for approximating fixed points of nonexpansive self-mappings defined on a Hilbert space. Fix $u \in C$, he studied the implicit iterative algorithm

$$
z_{t}=t u+(1-t) T z_{t}
$$

Namely, $z_{t}, t \in(0,1)$, is the unique fixed point of the contraction $x \mapsto t u+(1-t) T x, x \in C$. Browder proved that $\lim _{t \rightarrow+0} z_{t}=P u$, where $P u$ is the element of $F(T)$ nearest to $u$. Extensions to the framework of Banach spaces of Browder's convergence results have been done by many authors, including Reich [4], Takahashi and Ueda [5], and O'Hara et al. [6]. 
A family of mappings $\{T(t): t \geq 0\}$ is called a one-parameter strongly continuous semigroup of nonexpansive mappings (nonexpansive semigroup, for short) on $C$ if the following are satisfied.

(NS1) For each $t \geq 0, T(t)$ is a nonexpansive mapping on $C$.

(NS2) $T(s+t)=T(s) \circ T(t)$ for all $s, t \geq 0$.

(NS3) For each $x \in C$, the mapping $t \mapsto T(t) x$ from $[0, \infty)$ into $C$ is strongly continuous.

There are many papers concerning the existence of common fixed points of $\{T(t): t \geq 0\}$; see, for instance, [7-13]. As a matter of fact, Browder [8] proved that if $C$ is bounded, then $\bigcap_{t \geq 0} F(T(t))$ is nonempty.

Browder's type convergence theorem for nonexpansive semigroups is proved in [11, 14-18] and others. For example, the following theorem is proved in [17].

Theorem 1.1 (see [17]). Let $C$ be a closed convex subset of a Hilbert space E. Let $\{T(t): t \geq 0\}$ be a nonexpansive semigroup on $C$ such that $\bigcap_{t \geq 0} F(T(t)) \neq \emptyset$. Let $\left\{\alpha_{n}\right\}$ and $\left\{t_{n}\right\}$ be sequences in $\mathbb{R}$ satisfying

(C1) $0<\alpha_{n}<1$ and $0 \leq t_{n}$;

(C2) $\lim _{n} t_{n}=\lim _{n} \alpha_{n} / t_{n}=0$, where $1 / 0=\infty$.

Fix $u \in C$ and define a sequence $\left\{x_{n}\right\}$ in $C$ by

$$
x_{n}=\alpha_{n} u+\left(1-\alpha_{n}\right) T\left(t_{n}\right) x_{n} .
$$

Then $\left\{x_{n}\right\}$ converges strongly to the element of $\bigcap_{t \geq 0} F(T(t))$ nearest to $u$.

We note that $(\mathrm{C} 1)$ is needed to define $\left\{x_{n}\right\}$.

A nonexpansive semigroup $\{T(t): t \geq 0\}$ on $C$ is said to be uniformly asymptotically regular (u.a.r.) if for every $t \geq 0$ and for every bounded subset $K$ of $C$,

$$
\lim _{s \rightarrow \infty} \sup _{x \in K}\|T(s+t) x-T(s) x\|=0
$$

holds. The following is proved by Domínguez Benavides et al. [16]; see also [15].

Theorem 1.2 (see [16]). Let $E, C$, and $\{T(t): t \geq 0\}$ be as in Theorem 1.1. Assume that $\{T(t): t \geq$ $0\}$ is u.a.r. Let $\left\{\alpha_{n}\right\}$ and $\left\{t_{n}\right\}$ be sequences in $\mathbb{R}$ satisfying $(C 1)$ and

(D2) $\lim _{n} \alpha_{n}=0$ and $\lim _{n} t_{n}=\infty$.

Fix $u \in C$ and define a sequence $\left\{x_{n}\right\}$ in $C$ by (1.2). Then $\left\{x_{n}\right\}$ converges strongly to the element of $\bigcap_{t \geq 0} F(T(t))$ nearest to $u$.

There is an interesting difference between Theorems 1.1 and 1.2, that is, $\left\{t_{n}\right\}$ in Theorem 1.1 converges to 0 and $\left\{t_{n}\right\}$ in Theorem 1.2 diverges to $\infty$. By the way, very recently, Akiyama and Suzuki [14] generalized Theorem 1.1. They replaced (C2) of Theorem 1.1 by 
the following:

$\left(\mathrm{C} 2^{\prime}\right)\left\{t_{n}\right\}$ is bounded;

$\left(\mathrm{C}^{\prime}\right) \lim _{n} \alpha_{n} /\left(t_{n}-\tau\right)=0$ for all $\tau \in[0, \infty)$.

They also showed that the conjunction of $\left(\mathrm{C}^{\prime}\right)$ and $\left(\mathrm{C}^{\prime}\right)$ is best possible; see also [18].

In this paper, motivated by the previous considerations, we generalize Theorem 1.2 concerning $\left\{\alpha_{n}\right\}$ and $\left\{t_{n}\right\}$. Also, we will show that our new condition is best possible.

\section{Main Results}

We denote by $\mathbb{N}$ the set of all positive integers and by $\mathbb{R}$ the set of all real numbers. For $t \in \mathbb{R}$, we denote by $[t]$ the maximum integer not exceeding $t$.

The following proposition plays an important role in this paper.

Proposition 2.1. Let $C$ be a set of a separated topological vector space $E$. Let $\{T(t): t \geq 0\}$ be a family of mappings on $C$ such that $T(s) \circ T(t)=T(s+t)$ for all $s, t \in[0, \infty)$. Assume that $\{T(t): t \geq 0\}$ is asymptotic regular, that is,

$$
\lim _{s \rightarrow \infty}(T(t+s) x-T(s) x)=0
$$

for all $t \in[0, \infty)$ and $x \in C$. Then

$$
F(T(t))=\bigcap_{s \geq 0} F(T(s))
$$

holds for all $t \in(0, \infty)$.

Proof. Fix $t \in(0, \infty)$. It is obvious that $F(T(t)) \supset \bigcap_{s} F(T(s))$ holds. Let $z \in C$ be a fixed point of $T(t)$. For every $h \in[0, \infty)$, we have

$$
\begin{aligned}
T(h) z-z & =\lim _{n \rightarrow \infty}\left(T(h) \circ T(t)^{n} z-T(t)^{n} z\right) \\
& =\lim _{n \rightarrow \infty}(T(h+n t) z-T(n t) z) \\
& =\lim _{s \rightarrow \infty}(T(h+s) z-T(s) z) \\
& =0,
\end{aligned}
$$

and hence $z$ is a common fixed point of $\{T(t): t \geq 0\}$.

It is well known that every Hilbert space has the Opial property.

Proposition 2.2 (Opial [19]). Let $E$ be a Hilbert space. Let $\left\{x_{n}\right\}$ be a sequence in $E$ converging weakly to $z_{0} \in H$. Then the inequality $\lim _{\inf }\left\|x_{n}-z\right\| \leq \liminf _{n}\left\|x_{n}-z_{0}\right\|$ implies $z=z_{0}$.

We generalize Theorem 1.2. 
Theorem 2.3. Let $C$ be a closed convex subset of a Hilbert space E. Let $\{T(t): t \geq 0\}$ be a u.a.r. nonexpansive semigroup on $C$ such that $\bigcap_{t \geq 0} F(T(t)) \neq \emptyset$. Let $\left\{\alpha_{n}\right\}$ and $\left\{t_{n}\right\}$ be sequences in $\mathbb{R}$ satisfying (C1) and

$\left(\mathrm{D} 2^{\prime}\right) \lim _{n} \alpha_{n}=\lim _{n} \alpha_{n} / t_{n}=0$.

Fix $u \in C$ and define a sequence $\left\{x_{n}\right\}$ in $C$ by (1.2). Then $\left\{x_{n}\right\}$ converges strongly to the element of $\bigcap_{t \geq 0} F(T(t))$ nearest to $u$.

Proof. Put $F(\tau)=\bigcap_{t \geq 0} F(T(t))$. Let $v$ be the element of $F(\tau)$ nearest to $u$. Since

$$
\begin{aligned}
\left\|x_{n}-v\right\| & =\left\|\left(1-\alpha_{n}\right) T\left(t_{n}\right) x_{n}+\alpha_{n} u-v\right\| \\
& \leq\left(1-\alpha_{n}\right)\left\|T\left(t_{n}\right) x_{n}-v\right\|+\alpha_{n}\|u-v\| \\
& \leq\left(1-\alpha_{n}\right)\left\|x_{n}-v\right\|+\alpha_{n}\|u-v\|,
\end{aligned}
$$

we have $\left\|x_{n}-v\right\| \leq\|u-v\|$. Therefore $\left\{x_{n}\right\}$ is bounded. Hence $\left\{T(t) x_{n}: n \in \mathbb{N}, t \geq 0\right\}$ is also bounded.

We put

$$
M:=\sup \left\{\left\|T(t) x_{n}-u\right\|: n \in \mathbb{N}, t \geq 0\right\}<\infty .
$$

Let $\{f(n)\}$ be an arbitrary subsequence of $\{n\}$. Then there exists a subsequence $\{g(n)\}$ of $\{n\}$ such that $\left\{x_{f \circ g(n)}\right\}$ converges weakly to $x$. We choose a subsequence $\{h(n)\}$ of $\{n\}$ such that

$$
\tau:=\lim _{n \rightarrow \infty} t_{f \circ g \circ h(n)}=\limsup _{n \rightarrow \infty} t_{f \circ g(n)}
$$

Put $y_{j}=x_{f \circ g \circ h(j)}, \beta_{j}=\alpha_{f \circ g \circ h(j)}$, and $s_{j}=t_{f \circ g \circ h(j)}$. We will show $x \in F(\tau)$, dividing the following three cases:
(i) $\tau=\infty$,
(ii) $0<\tau<\infty$,
(iii) $\tau=0$.

In the first case, we fix $t \geq 0$. For sufficiently large $j \in \mathbb{N}$, we have

$$
\begin{aligned}
\left\|T(t) x-y_{j}\right\| & \leq\left\|T(t) x-T(t) y_{j}\right\|+\left\|T(t) y_{j}-y_{j}\right\| \\
& \leq\left\|x-y_{j}\right\|+\beta_{j}\left\|T(t) y_{j}-u\right\|+\left(1-\beta_{j}\right)\left\|T(t) y_{j}-T\left(s_{j}\right) y_{j}\right\| \\
& \leq\left\|x-y_{j}\right\|+\beta_{j} M+\left(1-\beta_{j}\right)\left\|T\left(s_{j}-t\right) y_{j}-y_{j}\right\| \\
& \leq\left\|x-y_{j}\right\|+\beta_{j} M+\left(1-\beta_{j}\right) \beta_{j}\left\|T\left(s_{j}-t\right) y_{j}-u\right\|+\left(1-\beta_{j}\right)^{2}\left\|T\left(s_{j}-t\right) y_{j}-T\left(s_{j}\right) y_{j}\right\| \\
& \leq\left\|x-y_{j}\right\|+\beta_{j}\left(2-\beta_{j}\right) M+\left(1-\beta_{j}\right)^{2}\left\|T\left(s_{j}-t+t\right) y_{j}-T\left(s_{j}-t\right) y_{j}\right\|,
\end{aligned}
$$


and hence

$$
\liminf _{j \rightarrow \infty}\left\|T(t) x-y_{j}\right\| \leq \liminf _{j \rightarrow \infty}\left\|x-y_{j}\right\|
$$

By the Opial property, we obtain $T(t) x=x$. Thus $x \in F(\tau)$.

In the second case, we have

$$
\begin{aligned}
\left\|T(\tau) x-y_{j}\right\| & \leq\left\|T(\tau) x-T\left(s_{j}\right) x\right\|+\left\|T\left(s_{j}\right) x-T\left(s_{j}\right) y_{j}\right\|+\left\|T\left(s_{j}\right) y_{j}-y_{j}\right\| \\
& \leq\left\|T(\tau) x-T\left(s_{j}\right) x\right\|+\left\|x-y_{j}\right\|+\beta_{j}\left\|T\left(s_{j}\right) y_{j}-u\right\| \\
& \leq\left\|T\left(\left|\tau-s_{j}\right|\right) x-T(0) x\right\|+\left\|x-y_{j}\right\|+\beta_{j} M,
\end{aligned}
$$

and hence

$$
\liminf _{j \rightarrow \infty}\left\|T(\tau) x-y_{j}\right\| \leq \liminf _{j \rightarrow \infty}\left\|x-y_{j}\right\|
$$

By the Opial property, we obtain $T(\tau) x=x$. By Proposition 2.1, we obtain $x \in F(\tau)$.

In the third case, we fix $t \geq 0$. For sufficiently large $j \in \mathbb{N}$, we have

$$
\begin{aligned}
\left\|T(t) x-y_{j}\right\| \leq & \left\|T(t) x-T\left(\left[t / s_{j}\right] s_{j}\right) x\right\|+\left\|T\left(\left[t / s_{j}\right] s_{j}\right) x-T\left(\left[t / s_{j}\right] s_{j}\right) y_{j}\right\| \\
& +\sum_{k=0}^{\left[t / s_{j}\right]-1}\left\|T\left(k s_{j}\right) y_{j}-T\left((k+1) s_{j}\right) y_{j}\right\|+\left\|T(0) y_{j}-y_{j}\right\| \\
\leq & \left\|T\left(t-\left[t / s_{j}\right] s_{j}\right) x-T(0) x\right\|+\left\|x-y_{j}\right\| \\
& +\left[t / s_{j}\right]\left\|T\left(s_{j}\right) y_{j}-y_{j}\right\|+\left\|T(0) y_{j}-T\left(s_{j}\right) y_{j}\right\|+\left\|T\left(s_{j}\right) y_{j}-y_{j}\right\| \\
\leq & \left\|T\left(t-\left[t / s_{j}\right] s_{j}\right) x-T(0) x\right\|+\left\|x-y_{j}\right\| \\
& +\left[t / s_{j}\right]\left\|T\left(s_{j}\right) y_{j}-y_{j}\right\|+\left\|y_{j}-T\left(s_{j}\right) y_{j}\right\|+\left\|T\left(s_{j}\right) y_{j}-y_{j}\right\| \\
= & \left\|T\left(t-\left[t / s_{j}\right] s_{j}\right) x-T(0) x\right\|+\left\|x-y_{j}\right\|+\left(\left[t / s_{j}\right]+2\right)\left\|T\left(s_{j}\right) y_{j}-y_{j}\right\| \\
= & \left\|T\left(t-\left[t / s_{j}\right] s_{j}\right) x-T(0) x\right\|+\left\|x-y_{j}\right\|+\left(\left[t / s_{j}\right]+2\right) \beta_{j}\left\|T\left(s_{j}\right) y_{j}-u\right\| \\
\leq & \max \left\{\|T(s) x-T(0) x\|: 0 \leq s \leq s_{j}\right\}+\left\|x-y_{j}\right\|+\left(t \beta_{j} / s_{j}+2 \beta_{j}\right) M .
\end{aligned}
$$

Hence (2.8) holds. Thus we obtain $x \in F(\tau)$.

We next prove that $\left\{y_{j}\right\}$ converges strongly to $v$. Since

$$
\begin{aligned}
& \beta_{j}\left\|y_{j}-v\right\|^{2}+\left(1-\beta_{j}\right)\left\langle\left(y_{j}-T\left(s_{j}\right) y_{j}\right)-\left(v-T\left(s_{j}\right) v\right), y_{j}-v\right\rangle \\
& \quad=\beta_{j}\left\langle u-v, y_{j}-v\right\rangle \\
& \left\langle\left(y_{j}-T\left(s_{j}\right) y_{j}\right)-\left(v-T\left(s_{j}\right) v\right), y_{j}-v\right\rangle \\
& \quad \geq\left\|y_{j}-v\right\|^{2}-\left\|T\left(s_{j}\right) y_{j}-T\left(s_{j}\right) v\right\|\left\|y_{j}-v\right\| \geq 0,
\end{aligned}
$$


we obtain $\left\|y_{j}-v\right\|^{2} \leq\left\langle u-v, y_{j}-v\right\rangle$. Since $\langle u-v, x-v\rangle \leq 0$, we have

$$
\begin{aligned}
\left\|y_{j}-v\right\|^{2} & \leq\left\langle u-v, y_{j}-v\right\rangle \\
& =\left\langle u-v, y_{j}-x\right\rangle+\langle u-v, x-v\rangle \\
& \leq\left\langle u-v, y_{j}-x\right\rangle,
\end{aligned}
$$

and hence $\left\{y_{j}\right\}$ converges strongly to $v$. Since $\left\{x_{f(n)}\right\}$ is arbitrary, we obtain that $\left\{x_{n}\right\}$ converges strongly to $v$. see [21].

Using [20, Theorem 7], we obtain the following Moudafi's type convergence theorem;

Corollary 2.4. Let $E, C,\{T(t): t \geq 0\},\left\{\alpha_{n}\right\}$, and $\left\{t_{n}\right\}$ be as in Theorem 2.3. Let $\Phi$ be a contraction on $C$; that is, there exists $r \in[0,1)$ such that $\|\Phi x-\Phi y\| \leq r\|x-y\|$ for $x, y \in C$. Define a sequence $\left\{x_{n}\right\}$ in $C$ by

$$
x_{n}=\alpha_{n} \Phi x_{n}+\left(1-\alpha_{n}\right) T\left(t_{n}\right) x_{n} .
$$

Then $\left\{x_{n}\right\}$ converges strongly to the unique point $z \in C$ satisfying $P \circ \Phi z=z$, where $P$ is the metric projection from $C$ onto $\bigcap_{t \geq 0} F(T(t))$.

We will show that $\left(\mathrm{D} 2^{\prime}\right)$ is best possible.

Example 2.5. Put $E=\ell^{2}(\mathbb{N})$, that is, $E$ is a Hilbert space consisting of all the functions $x$ from $\mathbb{N}$ into $\mathbb{R}$ satisfying $\sum_{k \in \mathbb{N}}|x(k)|^{2}<\infty$ with inner product $\langle x, y\rangle=\sum_{k \in \mathbb{N}} x(k) y(k)$. Define a bounded closed convex subset $C$ of $E$ by

$$
C=\left\{x \in E: 0 \leq x(k) \leq p_{k}\right\}
$$

where $p_{k}=2^{-k / 2}$. Define a u.a.r. nonexpansive semigroup $\{T(t): t \geq 0\}$ on $C$ by

$$
(T(t) x)(k)=\max \left\{x(k)-t p_{k}{ }^{2}, 0\right\} .
$$

Let $\left\{e_{k}\right\}$ be the canonical basis of $E$ and put $u=\sum_{k=1}^{\infty} p_{k} e_{k}$. Let $\left\{\alpha_{n}\right\}$ and $\left\{t_{n}\right\}$ be sequences in $\mathbb{R}$ satisfying (C1) and define $\left\{x_{n}\right\}$ in $C$ by (1.2). Then $\left\{x_{n}\right\}$ converges to a common fixed point of $\{T(t): t \geq 0\}$ only if $\lim _{n} \alpha_{n}=\lim _{n} \alpha_{n} / t_{n}=0$.

Proof. For $\alpha \in(0,1)$ and $t \geq 0$, we define $x(\alpha, t)$ by

$$
x(\alpha, t)=\alpha u+(1-\alpha) T(t) x(\alpha, t) .
$$


We note

$$
x(\alpha, t)(k)= \begin{cases}\alpha p_{k}, & \text { if } \alpha \leq t p_{k} \\ \left(1+t p_{k}-\frac{t p_{k}}{\alpha}\right) p_{k}, & \text { if } \alpha \geq t p_{k} .\end{cases}
$$

So, $x(\alpha, t)(k) \geq \alpha p_{k}$. It is obvious that $\bigcap_{t \geq 0} F(T(t))=\{0\}$. We assume $\lim _{n} x_{n}=\lim _{n} x\left(\alpha_{n}, t_{n}\right)=$ $P u=0$. Then

$$
0=\lim _{n \rightarrow \infty} \frac{x_{n}(1)}{p_{1}} \geq \lim _{n \rightarrow \infty} \alpha_{n}
$$

Arguing by contradiction, we assume $\lim \sup _{n} \alpha_{n} / t_{n}>0$. Then there exist $\kappa \in \mathbb{N}$ and a subsequence $\{f(n)\}$ of $\{n\}$ such that

$$
\frac{\alpha_{f(n)}}{t_{f(n)}} \geq 2 p_{\kappa}
$$

Since $\lim _{n} x_{f(n)}(\kappa)=0$, we have

$$
\begin{aligned}
0 & =\lim _{n \rightarrow \infty} \frac{x_{f(n)}(\kappa)}{p_{\kappa}}=\lim _{n \rightarrow \infty}\left(1+t_{f(n)} p_{\kappa}-\frac{t_{f(n)} p_{\kappa}}{\alpha_{f(n)}}\right) \\
& \geq \limsup _{n \rightarrow \infty}\left(1-\frac{t_{f(n)} p_{\kappa}}{\alpha_{f(n)}}\right) \geq \frac{1}{2}>0,
\end{aligned}
$$

which is a contradiction. Therefore we obtain $\lim _{n} \alpha_{n} / t_{n}=0$.

By Theorem 2.3 and Example 2.5, we obtain the following.

Theorem 2.6. Let $E$ be an infinite-dimensional Hilbert space. Let $\left\{\alpha_{n}\right\}$ and $\left\{t_{n}\right\}$ be sequences in $\mathbb{R}$ satisfying (C1). Then the following are equivalent:

(i) $\lim _{n} \alpha_{n}=\lim _{n} \alpha_{n} / t_{n}=0$,

(ii) if $C$ is a bounded closed convex subset $C$ of $E,\{T(t): t \geq 0\}$ is a u.a.r. nonexpansive semigroup on $C, u \in C$, and $\left\{x_{n}\right\}$ is a sequence in $C$ defined by (1.2), then $\left\{x_{n}\right\}$ converges strongly to the element of $\bigcap_{t \geq 0} F(T(t))$ nearest to $u$.

Compare $\left(\mathrm{D} 2^{\prime}\right)$ with the conjunction of $\left(\mathrm{C}^{\prime}\right)$ and $\left(\mathrm{C}^{\prime}\right)$. We can tell that the difference between both conditions is u.a.r.

\section{Acknowledgments}

The first author was partially supported by DGES, Grant MTM2006-13997-C02-01 and Junta de Andalucía, Grant FQM-127. The second author is supported in part by Grants-in-Aid for Scientific Research from the Japanese Ministry of Education, Culture, Sports, Science and Technology. 


\section{References}

[1] F. E. Browder, "Fixed-point theorems for noncompact mappings in Hilbert space," Proceedings of the National Academy of Sciences of the United States of America, vol. 53, pp. 1272-1276, 1965.

[2] W. A. Kirk, "A fixed point theorem for mappings which do not increase distances," The American Mathematical Monthly, vol. 72, pp. 1004-1006, 1965.

[3] F. E. Browder, "Convergence of approximants to fixed points of nonexpansive non-linear mappings in Banach spaces," Archive for Rational Mechanics and Analysis, vol. 24, pp. 82-90, 1967.

[4] S. Reich, "Weak convergence theorems for nonexpansive mappings in Banach spaces," Journal of Mathematical Analysis and Applications, vol. 67, no. 2, pp. 274-276, 1979.

[5] W. Takahashi and Y. Ueda, "On Reich's strong convergence theorems for resolvents of accretive operators," Journal of Mathematical Analysis and Applications, vol. 104, no. 2, pp. 546-553, 1984.

[6] J. G. O'Hara, P. Pillay, and H.-K. Xu, "Iterative approaches to finding nearest common fixed points of nonexpansive mappings in Hilbert spaces," Nonlinear Analysis, vol. 54, no. 8, pp. 1417-1426, 2003.

[7] L. P. Belluce and W. A. Kirk, "Nonexpansive mappings and fixed-points in Banach spaces," Illinois Journal of Mathematics, vol. 11, pp. 474-479, 1967.

[8] F. E. Browder, "Nonexpansive nonlinear operators in a Banach space," Proceedings of the National Academy of Sciences of the United States of America, vol. 54, pp. 1041-1044, 1965.

[9] R. E. Bruck Jr., "A common fixed point theorem for a commuting family of nonexpansive mappings," Pacific Journal of Mathematics, vol. 53, pp. 59-71, 1974.

[10] R. DeMarr, "Common fixed points for commuting contraction mappings," Pacific Journal of Mathematics, vol. 13, pp. 1139-1141, 1963.

[11] T. C. Lim, "A fixed point theorem for families on nonexpansive mappings," Pacific Journal of Mathematics, vol. 53, pp. 487-493, 1974.

[12] T. Suzuki, "Common fixed points of one-parameter nonexpansive semigroups," The Bulletin of the London Mathematical Society, vol. 38, no. 6, pp. 1009-1018, 2006.

[13] T. Suzuki, "Fixed point property for nonexpansive mappings versus that for nonexpansive semigroups," Nonlinear Analysis: Theory, Methods \& Applications, vol. 70, no. 9, pp. 3358-3361, 2009.

[14] S. Akiyama and T. Suzuki, "Browder's convergence for one-parameter nonexpansive semigroups," to appear in Canadian Mathematical Bulletin.

[15] A. Aleyner and Y. Censor, "Best approximation to common fixed points of a semigroup of nonexpansive operators," Journal of Nonlinear and Convex Analysis., vol. 6, no. 1, pp. 137-151, 2005.

[16] T. Domínguez Benavides, G. L. Acedo, and H.-K. Xu, "Construction of sunny nonexpansive retractions in Banach spaces," Bulletin of the Australian Mathematical Society, vol. 66, no. 1, pp. 9-16, 2002.

[17] T. Suzuki, "On strong convergence to common fixed points of nonexpansive semigroups in Hilbert spaces," Proceedings of the American Mathematical Society, vol. 131, no. 7, pp. 2133-2136, 2003.

[18] T. Suzuki, "Browder's type convergence theorems for one-parameter semigroups of nonexpansive mappings in Banach spaces," Israel Journal of Mathematics, vol. 157, no. 1, pp. 239-257, 2007.

[19] Z. Opial, "Weak convergence of the sequence of successive approximations for nonexpansive mappings," Bulletin of the American Mathematical Society, vol. 73, pp. 591-597, 1967.

[20] T. Suzuki, "Moudafi's viscosity approximations with Meir-Keeler contractions," Journal of Mathematical Analysis and Applications, vol. 325, no. 1, pp. 342-352, 2007.

[21] A. Moudafi, "Viscosity approximation methods for fixed-points problems," Journal of Mathematical Analysis and Applications, vol. 241, no. 1, pp. 46-55, 2000. 